\title{
Restrictions in Spatial Competition: The Effects on Firms and Consumers
}

\author{
Berno Buechel $^{1,2} \cdot$ Jan F. Klein ${ }^{3}$
}

Published online: 4 March 2016

(C) Springer International Publishing Switzerland 2016

\begin{abstract}
Despite the rich stream of research that evolved from Hotelling's spatial competition model, the fact that firms' strategies are constrained by their technological capabilities, the legal environment, or overriding corporate strategies is commonly neglected. We study a model of Hotelling-Downs competition in which two firms choose a position along a one-dimensional market given that their feasible positions are restricted to an interval. Strategy restrictions turn out to substantially affect firms' behavior and consumers' surplus. In contrast to existing results on spatial competition, we find that in equilibrium firms may minimally differentiate away from the center of the market or even locate completely independently of consumers' preferences. Assessing social welfare by total transportation costs, we observe that restrictions may both enhance and reduce welfare, depending on whether the feasible positions overlap.
\end{abstract}

We are thankful to Dinko Dimitrov, Saskia Fuchs, Esther Gal-Or, Gerd Muehlheusser, and Friedrich Roehrs for inspiring discussions and for helpful comments on earlier versions of this research note. A previous version of this paper circulated under the title "Do Consumers' Preferences Really Matter?-A Note on Spatial Competition with Restricted Strategies".

Jan F. Klein

jan.klein@aalto.fi

Berno Buechel

berno.buechel@unisg.ch;

http://www.berno.info

1 Institute of Economics (FGN), University of St. Gallen, Varnbüelstrasse 19, 9000 St., Gallen, Switzerland

2 Liechtenstein-Institute, Bendern, Liechtenstein

3 Department of Marketing, Aalto University School of Business, 21230, 0076 Aalto, Finland 
Keywords Product differentiation - Restrictions $\cdot$ Hotelling-Downs $\cdot$ Median voter · Duopoly

JEL Classification D43 $\cdot$ D49 $\cdot$ L13 $\cdot$ P16

\section{Introduction}

The design of new products is a key task for firms in virtually any industry. As an example, consider Samsung's tablet device called 'Galaxy' which is characterized by a certain size, screen resolution, and functionality. The 'patent war' between Samsung and Apple illustrates that the choice of these product specifications cannot be seen independently of Samsung's technological capabilities, which are further restricted by several patents on the competitor product 'iPad'. Generally, product specification choices have to account for (technological, legal, and other) constraints, for the competitor's products, and for the preferences of the consumers. While economic models of product differentiation incorporate heterogeneous consumer preferences and strategic behavior in the face of competition, they usually abstract from constraints of feasibility. Indeed, the models following the HotellingDowns approach of product differentiation focus on the effects of reducing fierce competition by increasing differentiation and on the effects of 'stealing' consumers of the competitor by decreasing differentiation. Thereby, they conceal that restrictions of the firm's and competitor's abilities affect strategic product positioning. This is unsatisfying-not only because empirical evidence documents that strategy restrictions are present when choosing a product position, but also because firms' behavior and consumers' surplus are substantially affected by strategy restrictions, as we will show in this note.

So far, when models predict that two firms choose similar product specifications with respect to one dimension (minimum differentiation), then the chosen position must be at the median of the distribution of consumers, which is interpreted as the center of the market or 'where the demand is'. In this note, we introduce restrictions of feasible positions in a model without price competition and observe that under various conditions, this conclusion does not hold true anymore. In particular, we find equilibria where the center of the market, would be a feasible product position for one firm, but this firm does not have an incentive to locate there, due to strategy restrictions of its main competitor. This suggests that the standardization of products, e.g. the similarity of Samsung's and Apple's tablets, does not inevitably result from consumers' tastes, but could also reflect a technological constraint of one of the two firms. In that case strategy restrictions reduce social welfare, while in another novel case that we study strategy restrictions contribute to product variety and thereby increase social welfare.

It is empirically well-justified to consider strategy restrictions because they appear to be ubiquitous in applications of horizontal differentiation. Restrictions have several sources and take many forms. In addition to technological constraints and the legal environment (e.g. patents, regulations), overriding corporate strategies 
can reduce the set of feasible product positions for a firm. ${ }^{1}$ Models of spatial competition, however, commonly abstract from the fact that, at least in the short term, the set of feasible product positions is restricted. This yields our two research questions: how do strategy restrictions affect product positions of the firms (i.e. equilibrium locations)? What are the welfare implications of strategy restrictions? We address these questions by using the most basic model: two players simultaneously choose a position on a line, while for each of them only some interval of positions is feasible. We use this simple yet powerful model as a benchmark to make the arguments clear and outline several model extensions.

We find in the equilibrium analysis that there are three cases to distinguish. In one case we obtain the standard minimum differentiation result that both firms locate on the median of the distribution of consumers. In the second case, when the median position is feasible for one of the two firms, this firm does not choose it in equilibrium but locates next to the strategy restriction of its competitor. To our best knowledge, this outcome, two firms locating next to each other but not at the center of the market, is new to the literature. We assess welfare by aggregated transportation costs and show that this novel case is highly inefficient. Transportation costs are even larger than in the classic case of minimum differentiation (at the center of the market). In the final case firms minimally differentiate on the boundaries of their restrictions independently of the location of the median of the distribution of consumers. This independence means that consumers' preferences do not influence firms' decisions in any way. However, this case tends to induce highest welfare which also exceeds welfare under unrestricted competition. While equilibrium positions in each case can be characterized by some form of minimum differentiation, consumers' preferences only play a vital role in the first case. Thus, our results differ from previous findings in that preferences of consumers are less dominant in determining market outcomes when restrictions are present and in that the implications for welfare are ambivalent, as under restrictions welfare can be both lower and higher compared to unrestricted competition.

Hence, the contribution of this note is threefold. First, we close a gap in the literature on spatial competition by providing conditions when and how strategy restrictions affect equilibrium outcomes. Already 30 years ago, Larry Samuelson pointed to this gap by noting that "the common assumption that candidates choose freely from the entire strategy space is an unrealistic one" (cf. Samuelson 1984). However, the effect of strategy restrictions on equilibrium locations has not been systematically discussed. Second, we demonstrate the significance of restrictions of feasible strategies for horizontal product differentiation. We show that under restrictions qualitatively different strategies become prevalent, where not the consumer, but the competitor is the first interest. As a result, restrictions are an essential factor for market outcomes. Third, we derive implications for consumers and a social planner by assessing social welfare. For instance, our results imply that if a social planner were in the position to impose restrictions on one firm, e.g. in

\footnotetext{
1 Specific examples for spatial restrictions in the literal sense are territorial restrictions in distribution, such as in the case of franchising (Stern et al. 1976), and zoning restrictions imposed by a government (Datta and Sudhir 2013). In the application of flight scheduling in the airline market (e.g. Panzar 1979; Schipper et al. 2007) strategies are restricted, e.g. by local bans on nighttime flying.
} 
order to increase product variety in a minimally differentiated market, it should only do so if it can impose restrictions on the other firm as well. Moreover, our model of spatial competition with restricted strategies does not only apply to firms in a market but also to parties in a political competition.

\subsection{Related Literature}

The Hotelling-Downs approach of spatial competition is a widely used tool in the analysis of product differentiation and of political competition. Models in this literature can generally be organized into location-cum-prices models which study a game of location choice before price competition (e.g. Hotelling 1929; d'Aspremont et al. 1979; de Palma 1985; Meagher and Zauner 2004; Król 2012; Tabuchi 2012) and purely spatial models (e.g. Downs 1957b; Eaton and Lipsey 1975; Prescott and Visscher 1977; Loertscher and Muehlheusser 2011) which abstract from endogenous price setting. A well-known result for location-cum-prices models is that firms maximally differentiate in a one-dimensional market. This holds in particular for a uniform distribution of consumers with quadratic transportation costs-a specification that avoids issues of non-existence (d'Aspremont et al. 1979). ${ }^{2}$ Generally, whether maximum differentiation occurs depends on a number of assumptions, including the distribution of consumers (e.g. Tabuchi and Thisse 1995; Anderson et al. 1997), the convexity of transportation cost (e.g. Economides 1986; Osborne and Pitchik 1987), the elasticity of demand (Böckem 1994; Xefteris 2013), and the presence of an unobserved dimension (Rhee et al. 1992). A caveat of location-cumprices models is that they often have to ensure existence of equilibria by assuming rather simple distributions of consumers and specific functional forms.

This is not true to the same extent for pure spatial models. Pure spatial models are not only sensible in regulated markets where prices are fixed, but also apply to markets in which prices are not a dominant marketing instrument. The latter occurs typically in twosided markets (Rochet and Tirole 2003; Armstrong 2006) such as a market for newspapers or media markets in general (George and Waldfogel 2006; Gal-Or and Dukes 2003). Pure spatial models typically find minimum differentiation under general conditions (Downs 1957b; Eaton and Lipsey 1975), but not when an endogenous number of players sequentially chooses a position (Prescott and Visscher 1977; Loertscher and Muehlheusser 2011). The discourse on maximum versus minimum differentiation has recently caught new fire with novel theoretical insights (Xefteris 2013) and with empirical evidence from the laboratory (Barreda-Tarrazona et al. 2011).

Considering the vast amount of literature dealing with the Hotelling-Downs approach, it is remarkable that the idea of restricting the firms' strategies plays hardly any role. Exceptions are the models of Samuelson (1984), Hummel (2010), and Hauser (1988). Samuelson (1984) analyzes a multi-dimensional model of probabilistic voting and incorporates restricted strategies in the following way: each candidate is endowed with an initial position and her choice of strategy is restricted

\footnotetext{
2 In a multi-dimensional market, the same specification leads to maximum differentiation with respect to one dimension and to minimum differentiation with respect to all others (Irmen and Thisse 1998). Moreover, using an evolutionary approach Hehenkamp and Wambach (2010) show that minimum differentiation with respect to all dimensions emerges in this setting.
} 
within some convex compact set around this endowment. We follow this approach on how to restrict positions. In the context of brand positions Hauser (1988) introduces a restriction on feasible positions by assuming that given positions cannot change their relative order. A similar assumption is made by Hummel (2010) in the context of political candidates since his model restricts political candidates from moving more than halfway into the direction of the opponent. ${ }^{3}$ Within the context of political competition there are additional approaches to incorporate restrictions of positions (see Samuelson 1984, and the references therein). Even though these works use the important idea of strategy restrictions, they do not systematically discuss the effect of restrictions on equilibrium locations and they focus on the application to political competition, but not to product differentiation.

Introducing strategy restrictions typically does not qualitatively affect maximum differentiation results. For example, in the very common location-cum-price model of uniformly distributed consumers facing quadratic transportation costs (d'Aspremont et al. 1979), strategy restrictions would not change the outcome, apart from the fact that firms can only maximally differentiate within their feasible sets. Minimum differentiation results, on the other hand, have to be carefully reconsidered, as we will discuss in the following.

\section{A Model with Restricted Strategies}

We define a game between two players $L$ and $R$, who compete in a one-dimensional market. The players simultaneously choose a product position in order to maximize their payoff.

There is a continuum of positions $X=[0,1]$. Positions are ordered by the relation $\leq$ such that we can refer to absolute distances $(|x-y|)$, being closer $(\mid x-$ $y|<| x-z \mid)$ or being in between $(x<y<z)$. We consider a unit mass of consumers who are distributed on $X$ according to the cumulative distribution function $F: X \rightarrow$ $R_{+}$with full support in the corresponding density function: $f(x)>0$ for any $x \in X$. Let $q:=F^{-1}\left(\frac{1}{2}\right) \in X$ be the median of the distribution, which is also referred to as the 'median consumer' (Waldfogel 2008, p. 568). ${ }^{4}$ If the distribution satisfies symmetry, i.e. $F(x)=1-F(1-x)$, then the median is $q=\frac{1}{2}$.

Consumers are distinguished from firms, or players, whom we denote by $L$ and $R .^{5}$ Because of technological or other constraints, players' strategies are restricted in the sense of Samuelson (1984), i.e. a strategy (product position) $s^{P}$ for $P=L, R$, cannot be chosen freely from all the product positions in $X$ but only from some compact convex subset.

Assumption We define the set of feasible product positions for each player as an interval within $X$, i.e. a player $P=L, R$ has the strategy set $S^{P}=\left[\underline{S}^{P}, S^{-P}\right]$

\footnotetext{
3 This is motivated by the idea that a change in political position from a primary to a general election, socalled 'flip-flopping', undermines a candidate's credibility.

4 Depending on the application the much more common term 'median voter' can be used synonymously.

5 As a convention we use the male form for consumers and the female form for players.
} 
Let $S=S^{L} \times S^{R}$ denote the strategy space and let $s^{-P}\left(S^{-P}\right)$ denote the strategy (set) of the player that is not $P$. Without loss of generality we let $\underline{S}^{L} \leq \underline{S}^{R}$.

We assume that the firms' costs are independent of the chosen product position and normalized to zero. Moreover, we abstract from price competition because it would lead to trivial results (under the common set-up), as discussed above. Thus, profit maximization in this model equals the maximization of market share. Consumers are assumed to buy one unit at the firm that is closer to them. Let $\hat{x}:=\frac{s^{L}+s^{R}}{2}$ be the position of an indifferent consumer. Then the players' payoffs for a strategy profile $s^{L}<s^{R}$ are as follows:

$$
\begin{gathered}
\pi^{L}(s)=F(\hat{x}), \\
\pi^{R}(s)=1-F(\hat{x})
\end{gathered}
$$

and vice versa for $s^{L}>s^{R}$. For two equal positions $s^{L}=s^{R}$ we assume that the two firms split the market equally, i.e. $\pi^{L}(s)=\pi^{R}(s)=\frac{1}{2}$. We solve our model with the standard notion of Nash equilibrium. In one case there will be an open set problem very similar to Bertrand competition with constant but unequal marginal costs. ${ }^{6} \mathrm{We}$ tackle this issue by studying epsilon-equilibria as done by Radner (1980) and Dixon (1987). ${ }^{7}$

Definition (cf. Dixon 1987). Let $\epsilon \geq 0$. $s \in S$ is a epsilon-equilibrium if for $P=L$, $R$, there does not exist a strategy $\tilde{s}^{P} \in S^{P}$ such that

$$
\pi^{P}\left(\tilde{s}^{P}, s^{-P}\right)-\pi^{P}(s)>\epsilon .
$$

Clearly, for sufficiently large $\epsilon$ every strategy profile is an epsilon-equilibrium. Thus, this notion is sensible for small epsilon only. In particular, for $E=0$ it coincides with the notion of Nash equilibrium.

\section{Equilibrium Positions}

To analyze our model we distinguish between three cases, which are distinct and exhaustive.

\subsection{Case (I)}

In Case (I) we analyze the model when the feasible strategies overlap and the median is part of this intersection, i.e. $q \in S^{L} \cap S^{R}$. An example for Case (I) is $S^{L}=S^{R}=X$, the classic model of unrestricted strategies. The result of Black (1948) on majority voting implies that the equilibrium outcome in this location

\footnotetext{
6 In Bertrand competition there does not exist a smallest price difference to undercut an opponent. In our model there will not exist a smallest unit of product differentiation.

7 Other ways to handle the open set issue would not lead to qualitatively different results.
} 


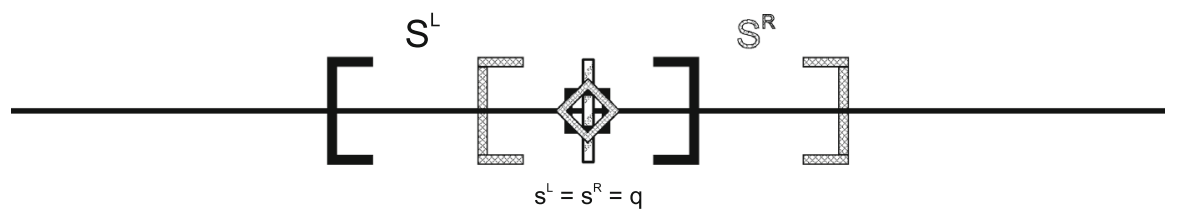

Fig. 1 In Case (I), i.e. $q \in S^{L} \cap S^{R}$, both players choose the median as their product position: $s^{L}=s^{R}=q$. This result equals the result of unrestricted competition

game is minimum differentiation on the median as illustrated in Fig. 1. Because this is an equilibrium in the classic model of unrestricted strategies, it must also be an equilibrium in our model, since the set of possible deviations has been reduced. Our first result establishes that there are no additional equilibria.

Proposition 1 If $q \in S^{L} \cap S^{R}$, the unique Nash-equilibrium is that both players choose the median as their product position, i.e. $s^{L}=s^{R}=q$.

Proof By the definition of the median $q$ we have $F(q)=1-F(q)=\frac{1}{2}$. Suppose $s$ is such that no player chooses the median $q$. Let $P$ be a player with $\pi^{P}(s) \leq \pi^{-P}(s)$. Deviating to $\tilde{s}^{P}=q$ leads to a payoff of $\pi^{P}\left(q, s^{-P}\right)>\frac{1}{2}$ while $\pi^{P}(s) \leq \frac{1}{2}$. Suppose $s$ is such that exactly one player, $P$, chooses the median. Then $\pi^{-P}(s)<\frac{1}{2}$, while $\pi^{P}(q, q)=\frac{1}{2}$. Finally, for $s^{L}=s^{R}=q$ any deviation leads to a lower payoff.

In Case (I) we get the classic outcome of minimum differentiation (on the median), despite strategy restrictions.

As a practical example for this case we can consider the market for credit cards since neither MasterCard nor Visa seems to be hindered from offering a product at the center of the market. Consequently, the services offered by these two firms should be highly similar.

Minimum differentiation is known to be inefficient since the work of Hotelling (1929). Given minimum differentiation, the median is the position that minimizes the sum of distances to all consumers. In that sense the median incorporates the preferences of the consumers and locating on the median is constrained efficient. Comparative-static changes in preferences or changes in the sample of consumers would affect the location of the median and thus the product positions taken by the players, as long as the condition $q \in S^{L} \cap S^{R}$ still holds, i.e. as long as we are still in Case (I).

\subsection{Case (II)}

In Case (II) we examine the situation where the feasible strategies $S^{L}$ and $S^{R}$ overlap and the median is not part of the intersection, i.e. $S^{L} \cap S^{R} \neq \emptyset$ and $q \notin S^{L} \cap S^{R}$.

Definition 1 (More-central player). If there is a player who can choose a product position which is strictly closer to the median, she is called the more-central player (MC); her opponent is called the less-central player (LC). 
In Case (II) we have to distinguish between two different subcases: in Subcase (IIa) a more-central player does not exist and in Subcase (IIb) a more-central player exists. Subcase (IIa) is given if the players' feasible product positions share a boundary and the median is beyond this boundary, e.g. $q<\underline{s}^{L}=\underline{s}^{R}$. This case occurs when there is a technological or legal restriction that hinders both firms in the same way from offering a product at the center of the market. In Subcase (IIb) the median is not in the strategy set of the less-central player, e.g. $q<\underline{s}^{L C}$, while it might, but need not, be in the strategy set of the more-central player, i.e. $\underline{s}^{M C}<\underline{s}^{L C}$. A particular example for this subcase is given if one player is unrestricted, i.e. $S^{P}=X$, while the other player is restricted such that she cannot choose the median $q \notin S^{-P}$.

Figure 2 illustrates the two subcases and the corresponding equilibrium analysis. The strategic situation resembles Bertrand competition with constant marginal costs. In the Bertrand model players can improve by undercutting the opponent's prices for any price above the marginal costs; here players can improve by locating closer to the median for any strategy away from the restriction boundary. In the Bertrand model we have a unique Nash equilibrium for equal marginal costs and only epsilon-equilibria under unequal marginal costs. Here, we find a fully analogous result in Subcase (IIa) and an analogous, but a unique type of epsilonequilibrium in Subcase (IIb), as Proposition 2 shows.

Proposition 2 Suppose $S^{L} \cap S^{R} \neq \emptyset$ and $q \notin S^{L} \cap S^{R}$.

(i) If a more-central player does not exist, the unique Nash equilibrium is such that both players take a product position on the edge of their restriction toward the median, i.e. if $q<\underline{s}^{L}=\underline{s}^{R}$, then $s=\left(\underline{s}^{L}, \underline{s}^{R}\right)$ is the Nash equilibrium; if $q>\bar{s}^{L}=\bar{s}^{R}$, then $s=\left(\bar{s}^{L}, \bar{s}^{R}\right)$ is the Nash equilibrium.

(ii) If a more-central player exists, then for any $E>0$, there is an E-equilibrium such that the less-central player takes the product position on the edge of her restriction in direction of the median and the more-central player locates closely next to it in direction of the median, i.e. if $\underline{s}^{M C}<\underline{s}^{L C}$ and

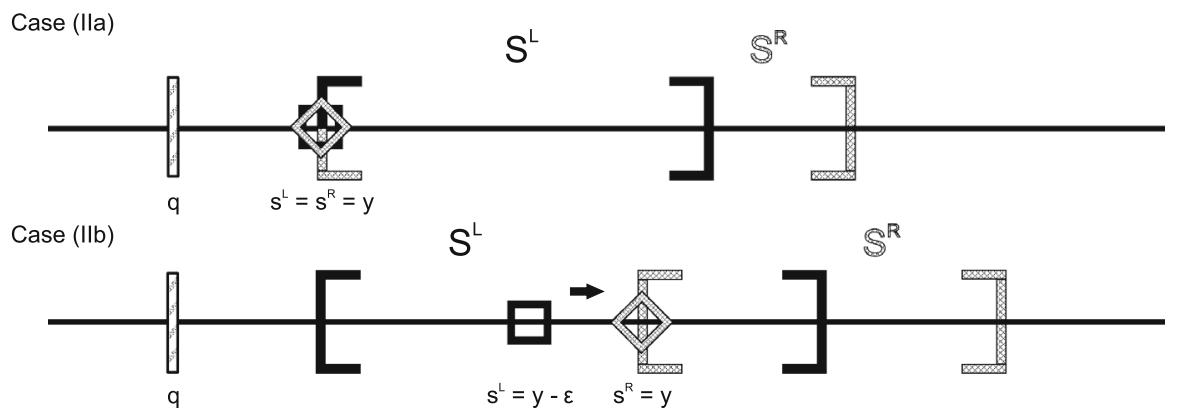

Fig. 2 Case (IIa) if a more-central player does not exist, i.e. none of the players can choose a product position closer to the median than her opponent, both players take the same product position on the edge of their restriction toward the median. Case (IIb) if a more- central player exists (here: $L$ ), the less-central player (here: $R$ ) locates on the edge of her restriction toward the median, while the more-central player approaches this position from the side of the median (here: from the left) 
$q<\underline{s}^{L C}$, then $s^{L C}=\underline{s}^{L C}$ and $s^{M C}=\underline{s}^{L C}-\varepsilon$; if $\bar{s}^{P}>\bar{s}^{-P}$ and $q>\bar{s}^{-P}$, then $s^{-P}=\bar{s}^{-P}$ and $s^{P}=\bar{s}^{-P}+\varepsilon$, for some $\varepsilon>0$.

\section{Proof}

(i) Let $y$ be the feasible position which is closest to the median. No player can improve by relocating from $s^{L}=s^{R}=y$ since relocation has to be in the opposite direction of the median. Now let $\hat{s} \neq s$ be a strategy profile where not both players locate on $y$. Take a player $P$ such that $\pi^{P}(\hat{s}) \leq \pi^{-P}(\hat{s})$ and relocate her to $\tilde{s}^{P}=y$. This is a strict improvement (irrespective of whether the other player $-P$ is also located on $y$ ).

(ii) Assume $\underline{s}^{L}<\underline{s}^{R}$ and $q<\underline{s}^{R}$ such that $L$ is the more-central player. Suppose $L$ locates within the feasible set of $R$, i.e. $s^{L} \in S^{R}$. If $\pi^{L}(s) \leq \frac{1}{2}, L$ can improve by moving closer to the median; if $\pi^{L}(s)>\frac{1}{2}, R$ can improve by choosing the same position as $L$. Thus, in equilibrium $s^{L}<\underline{s}^{R}$. Suppose $s^{R} /=\underline{s}^{R}$, then $R$ can improve by moving to $\underline{s}^{R}$. Thus, in equilibrium $s^{R}=\underline{s}^{R}$. The maximal possible payoff for $L$ is then bounded from above by $F\left(\underline{s}^{R}\right)$. Choosing $s^{L}=\underline{s}^{R}-\varepsilon$ leads to a payoff of $F\left(\underline{s}^{R}-\frac{\varepsilon}{2}\right)$. For small $\varepsilon$, any difference $E$ between these payoffs can be undercut. Thus, $\left(\underline{s}^{R}-\varepsilon, \underline{s}^{R}\right)$ is an $E$-equilibrium for sufficiently small $\varepsilon$. If $\bar{s}^{P}>\bar{s}^{-P}$ and $q>\bar{s}^{-P}$, then the proof is in full analogy to above.

As a practical example for Subcase (IIa) consider two neighboring countries, where a certain product is only legal in one of the two, such as it is the case for the United States and Mexico with respect to some medication. No firm can choose a location for a shop (e.g. a pharmacy) at the center of the market of the country where the product is illegal (e.g. the US). According to Proposition 2 part (i) each firm chooses a position at the common restriction (i.e. the border).

As a practical example for Subcase (IIb) we might consider the position of Mc-Donald's and Burger King restaurants on the dimension "tasty versus healthy" food. Arguably, by its history (in particular its previous marketing activities) Burger King is restricted from locating at the median position, which is more healthy than what Burger King can credible represent. If the restriction of McDonald's in this respect is not as tight, then McDonald's is the more-central player and thus approaches Burger King from the healthy side (according to Proposition 2 part (ii)). Consistent with reality, the positions of McDonald's and Burger King would not strongly differ, but McDonald's would be the clear market leader.

In Subcase (IIa) both players locate as closely as possible to the median, which is qualitatively similar to the standard result of Case (I). In Subcase (IIb) this only holds for the less-central player. For the more-central player, however, it is possible 
that the median is a feasible product position, but this central position will never be chosen. Instead, the more-central player minimally differentiates to the opponent's strategy restriction. The practical implication of this scenario is that products are sold which do not fit to the median consumer's taste, although this would be a feasible position for one of two firms. This is highly inefficient, as we will see in Sect. 3.4.

\subsection{Case (III)}

In the remaining Case (III) we examine the model with restricted strategies when the feasible strategies do not overlap, i.e. $S^{L} \cap S^{R}=\emptyset$.

Proposition 3 If $S^{L} \cap S^{R}=\emptyset$, then $s^{L}=\bar{s}^{L}$ and $s^{R}=\underline{\mathrm{s}}^{\mathrm{R}}$ are strictly dominant strategies.

Proof For any strategy profile with $s^{L}<\bar{s}^{L}$, changing to $\tilde{s}^{L}=\bar{s}^{L}$ is a strict improvement because it shifts the indifferent consumer $\hat{x}$ to the right. Analogously, for player $R$.

Proposition 3 shows that in the unique Nash equilibrium both players locate at the edge of their strategy set in direction of the opponent as illustrated in Fig. 3. While the result is trivial, it has a remarkable implication.

Unlike Case (I) and Case (II), equilibrium positions in Case (III) do not depend on the median. Even more generally, consumers preferences do not at all affect the position of the players, as noted in Remark 3.3.

Remark Equilibrium positions in Case (III) are independent of consumers' preferences (represented by $F$ or $q$ ).

Consequently, firms might offer products which do not respect consumers' needs. For instance, it is possible, as in Case (II), that the median position is available for one of the two players (such as in Fig. 3), but she does not choose it.

As a practical example for this case we suggest presidential elections in the United States. This example of political competition is often used to illustrate unrestricted spatial competition where both candidates choose the median position. It is, however, questionable to assume that strategies of a Republican and a Democratic candidate are unrestricted or to suppose-which is shown to be sufficient for the same outcome (c.f. Case (I)) - that feasible strategies

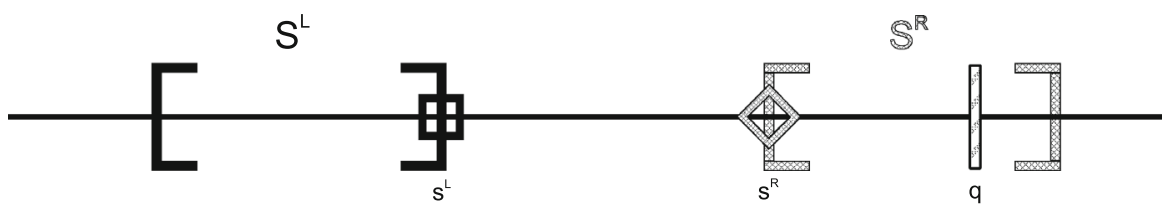

Fig. 3 Equilibrium positions in Case (III), i.e. if $S^{L} \cap S^{R}=\emptyset$. Both players locate as closely as possible to the opponent's strategy set. Equilibrium strategies are independent of the position of the median $q$ and thus independent of consumers' preferences 
really overlap at the median. It seems equally plausible to assume that feasible strategies of a Republican and a Democratic candidate do not overlap such that Case (III) applies. Then the presidential candidates choose a position as closely as possible toward the opponent without ending up at the same position.

\subsection{Welfare Implications}

In our model producers' surplus is constant because firms play a zero-sum-game for market share. Therefore, welfare effects can be discussed solely on the basis of the consumers' surplus, whose utility is decreasing in the individual costs of transportation. Welfare is hence negatively measured by the total transportation costs $T C\left(s^{L}, s^{R}\right)$, which is the sum of distances of each consumer to a closest player. ${ }^{8}$ Formally, for $s^{L} \leq s^{R}$,

$$
T C\left(s^{L}, s^{R}\right):=\int_{0}^{\hat{x}}\left|s^{L}-x\right| f(x) d x+\int_{\hat{x}}^{1}\left|s^{R}-x\right| f(x) d x
$$

To ease the exposition, let us assume here that consumers are uniformly distributed, i.e. $f(x)=1$ for all $x$. Then (2) simplifies to $T C\left(s^{L}, s^{R}\right)=\frac{3}{4}\left(s^{L}\right)^{2}+\frac{3}{4}\left(s^{R}\right)^{2}-\frac{1}{2} s^{L} s^{R}-s^{R}+\frac{1}{2}$. The global optimum is attained for $s^{L}=\frac{1}{4}$ and $s^{R}=\frac{3}{4}$, which yields $T C\left(\frac{1}{4}, \frac{3}{4}\right)=\frac{1}{8}^{9}$. We will use this social optimum as a benchmark to assess efficiency of the three cases. In particular, to quantify inefficiency we report the so-called Price of Anarchy (henceforth: PoA), which is attained by dividing the "worst" equilibrium, i.e. the equilibrium strategy profile with maximal transportation costs, by the globally minimal transportation costs (Koutsoupias and Papadimitriou 2009). ${ }^{10}$

In Case (I) both players locate on the median $q\left(=\frac{1}{2}\right)$. This yields total transportation costs of $T C(q, q)=\frac{1}{4}$ and, hence, a PoA of 2 .

In Subcase (IIa) both players choose the same location, say $y$, which was their common boundary in direction of the median. Since in Subcase (IIb) players differentiate only by some $\varepsilon$, which is vanishingly small for a notion of epsilonequilibrium close to the notion of Nash equilibrium, we can approximate welfare properties of this subcase also by equal positions $s^{L}=s^{R}=y$. In an extreme example strategies are restricted to be at an endpoint of the line, e.g.

\footnotetext{
${ }^{8}$ In models where transportation costs are quadratic in distance it is common to use the sum of squared distances (e.g. d'Aspremont et al. 1979; Meagher and Zauner 2004; Król 2012). Since our model is more general in this respect, we use linear transportation costs to measure efficiency. The choice of efficiency criterion, however, is not crucial for our discussion.

9 By the classic 'business stealing' argument, the efficient strategy profile is not an equilibrium if at least one player can move closer to the median.

${ }^{10}$ Likewise the Price of Stability refers to the "best" equilibrium in relation to the social optimum. There is no need to use both measures in our model since we have uniqueness of equilibria, up to small differences between a multitude of epsilon-equilibria in Subcase (IIb).
} 
$S^{L}=S^{R}=\{0\}$, which yields the globally maximal transportation costs of $\frac{1}{2}$. More generally, transportation costs are U-shaped in the common location $y$ and the closer $y$ to the median, the lower the transportation costs. Thus, in Case (II) the transportation costs lie in the interval $\left(\frac{1}{4}, \frac{1}{2}\right]$, which yields a PoA in the interval $(2$, 4]. The intervals are open on one side because the median $q$ cannot coincide with the common location $y$ when we are in Case (II).

The unique equilibrium in Case (III) is that players choose $s^{L}=\bar{s}^{L}$ and $s^{R}=\underline{s}^{R}$. By coincidence this choice may be socially optimal (which happens when $\bar{s}^{L}=\frac{1}{4}$ and $\underline{s}^{R}=\frac{3}{4}$ ), but there are also examples with almost maximal transportation costs, e.g. if $S^{L}=\{0\}$ and $S^{R}=\{0.001\}$. Therefore, in Case (III) the transportation costs lie in the interval $\left[\frac{1}{8}, \frac{1}{2}\right)$ with a corresponding PoA in the interval $[1,4)$. If we focus, however, on situations where the median is between the two strategy sets, i.e. $\bar{s}^{L}<q<\underline{s}^{R}$, e.g. because the players' restrictions are symmetric with respect to $q=\frac{1}{2}$, then inefficiency is bounded. The worst case example is then $S^{L}=\{0\}$ and $S^{L}=\{1\}$, which yields transportation costs of $\frac{1}{4}$ and a PoA of 2. Similarly, if we suppose that the difference between the two strategy sets is at least one third, i.e. $\underline{s}^{R}-\bar{s}^{L} \geq \frac{1}{3}$, the same conclusion holds. Table 1 summarizes the welfare properties of the three cases, where Case (III+) stands for Case (III) under the qualification that at least one of these two properties, either the median is between the restrictions or the restrictions differ by at least one third, holds. We observe that the three cases can be ranked according to the welfare they induce in equilibrium as follows:

$$
\text { Case }(\mathrm{III}+) \succeq \operatorname{Case}(\mathrm{I}) \succeq \operatorname{Case}(\mathrm{II}) \text {. }
$$

This holds with respect to the cardinal criterion of total transportation costs. Considering the ordinal notion of Pareto efficiency, we come to a similar conclusion. Equilibrium positions in Case (I) and Case (II) are not Pareto efficient, because one of the two minimally differentiated players could be relocated to shorten distances of some consumers without harming all others. On the other hand, equilibrium positions in Case (III) are Pareto efficient. We can summarize the welfare implications of strategy restrictions by comparing unrestricted strategies $S^{P}=X$ with restricted strategies $S^{P} \subset X$. Interestingly, unrestricted competition

Table 1 Summary of welfare properties of equilibria for different cases under the assumption of a uniform distribution of consumers

\begin{tabular}{llll}
\hline Case & Transportation costs & Price of anarchy & Market share \\
\hline Case (I) & $\frac{1}{4}$ & 2 & $50 \%$ \\
Case (IIa) & $\left(\frac{1}{4}, \frac{1}{2}\right]$ & $(2,4]$ & $50 \%$ \\
Case (IIb) & $\left(\frac{1}{4}, \frac{1}{2}\right]$ & $(2,4]$ & $(50-100 \%)$ \\
Case (III) & {$\left[\frac{1}{8}, \frac{1}{2}\right)$} & {$[1,4)$} & {$[50-100 \%)$} \\
Case (III+) & {$\left[\frac{1}{8}, \frac{1}{4}\right]$} & {$[1,2]$} & {$[50-75 \%]$} \\
\hline
\end{tabular}

Market share stands for the equilibrium market share of the larger competitor, which measures inequality between firms 
where all positions are feasible (which is a special example of Case (I)) leads to a worse outcome than Case (III+) that implies that restrictions are strong enough to exclude some positions from the feasible set of both firms. Moreover, if only one player is restricted, while the other can cover the entire spectrum (which is a special example of Case (II)), the outcome is even worse. Thus, if a social planner were in the position to impose a restrictions of one firm, it should only do so if it can impose a restriction on the other firm as well.

Let us finally discuss the consequences for firms. As mentioned above, for firms there is no issue of inefficiency in our model, but we can discuss their inequality. Market shares are equal in equilibrium in Case (I) and in Subcase (IIa). In Subcase (IIb), the more-central player receives a larger proportion of the consumers. Finally, in Case (III) player $L$ receives a higher payoff if and only if $\bar{s}^{L}$ is closer to the median than $\underline{s}^{R}$. These observations are also indicated in Table 1, where the last column reports the equilibrium market share of the largest competitor. Moreover, it can easily be shown that a player $P$ receives at least half of all consumers

(a) if her strategy set includes the median, i.e. $q \in S^{P}$, or

(b) if her strategy set is a superset of the opponent's, i.e. $S^{P} \supseteq S^{-P}$.

In that sense, a strategy set, e.g. a technology, is particularly valuable if (a) it allows to serve the center of the market and if (b) it is more flexible than the opponent's.

\section{Discussion}

We have introduced restrictions of feasible positions for two players who compete in a one-dimensional market. A striking insight is that in the case where feasible positions do not overlap (Case III) equilibrium choices are fully independent of consumers' preferences. Thus, in that case firms are predicted to ignore the consumers and base their product position on the competitor's strategy only. Still, welfare tends to be high since strategy restrictions contribute to product variety in this case. Another novel case emerges when one firm's feasible positions are more central than the other's while they still overlap (Case IIb). Then firms minimally differentiate away from the center of the market, which leads to highest transportation costs and thus to lowest welfare. Both these cases document that product positioning under strategy restrictions lead to qualitatively new insights, which we can observe even in this particularly simple model.

Our analysis naturally applies to short-term competition where product positions underlie fixed restrictions. In a longer term firms can be assumed to invest into changes of their feasible positions such that restrictions are endogenously determined. Since choosing the median guarantees a firm to receive half of the consumers (cf. Property (a) in Sect. 3.4), it seems that in a longer term, the standard result (of Case I) is prevalent. However, this fails to be true in several settings including the following two. First, consider an incumbent firm who is able to react 
to entrants and whose consumers do not switch to an entrant if both firms choose the same position. Then the optimal choice of restrictions of the incumbent is to span an interval at the center of the market. The entrant does not locate within the incumbent's feasible set, but approaches it from one side. ${ }^{11}$ After the incumbent's reaction the market outcome corresponds to Case (IIb), which leads to lowest welfare. Second, consider a firm investing into patents that protect a certain range of product specifications. The choice of a set of patents $P=[\underline{P}, \bar{P}]$ restricts the feasible strategies of a competitor to $X \backslash P=[0, \underline{P}) \cup(\bar{P}, 1]$. Given that the competitor has to first decide whether to approach the patent from the left or from the right, e.g. by developing the necessary technology, the patent holder can locate on the corresponding boundary of its patent and would choose a broad set of patents around the median in the first place. Again, the equilibrium outcome coincides with the outcome of Case (IIb).

The practical prediction of this theoretical outcome is that the firms choose highly similar product specifications, which are far from an ideal product in the eyes of most of the consumers. This holds despite the fact that a superior product would be feasible for one of the two firms, which is the more-central player, the incumbent, or the patent holder, in the respective model.

As many models of spatial competition, our model also applies to political competition. Let us now change the interpretation from firms and consumers to political candidates and voters. The left-right spectrum stands for possible political platforms from left-wing to right-wing positions. Political candidates also have a restricted set of feasible positions (Samuelson 1984) for two reasons. First, a candidate has to 'maintain a loyalty' to its political party. These parties only cover some part of the political spectrum, they need sponsors and supporters, and are relatively ideologically immobile (Downs 1957a). Second, the own history of public perception restricts the political positions that a candidate can credibly represent. A similar set of credibility restrictions is based on personal characteristics (Samuelson 1984), e.g. a young candidate cannot credibly present himself as highly experienced.

In the classic model the main result is often summarized by the role of the socalled median voter. At least two forms of the median voter theorem are popular (Congleton 2002). The weak form of the median voter theorem claims that the median voter always casts his vote for the policy that is adopted. As it is easy to show, this is still true in the model with restricted strategies. The strong form of the median voter theorem says that the median voter always gets his most preferred policy. With restricted strategies this is obviously not possible if $q \notin S^{L} \cup S^{R}$. However, even if the median position is available, it is not always chosen such as in Case (IIb) or in Case (III). Under unrestricted strategies this could not be an equilibrium because each candidate could beneficially deviate toward the median voter. If one candidate is incapable of such a move, however, the other candidate lacks incentive to do so. Thus, restrictions of political platforms do severely affect political campaigns and the outcome of two-party competition.

Our model uses several standard simplifications which have been relaxed for the classic model and are also worth studying under restricted strategies. First, the

11 A comprehensive and formal discussion of such a model can be requested from the authors. 
assumption of perfectly inelastic demand could be relaxed such as in Anderson and Glomm (1992) and George and Waldfogel (2006). Second, we have studied a onedimensional market. A natural extension is to consider multi-dimensional product differentiation. In such a model Irmen and Thisse (1998) find that minimum differentiation is prevalent in all dimensions but one. While in each dimension with minimum differentiation both firms cluster on the median, our analysis indicates that this result would not generalize to restricted strategies. Thus, our onedimensional analysis represents the first step to understand multi-dimensional competition. Nevertheless, additional strategic effects may emerge in multidimensional competition with strategy restrictions. Another important extension is to consider larger classes of consumers', respectively voters', preferences. Our results do directly extend to single-peaked preferences on tree graphs (Demange 1982), which is a much more general class of preferences. However, the extension to graphs that include cycles, e.g. grids or hypercubes, as they have been studied in Nehring and Puppe (2007) or Buechel and Roehl (2015), is an open problem. Finally, we have restricted attention to the classic case of two players. While for an exogenous number of players, the results under unconstrained competition resemble the two player case ("minimum clustering," Eaton and Lipsey 1975), this is not true for an endogenous number of players (Prescott and Visscher 1977; Loertscher and Muehlheusser 2011). Extending our model into these directions is left for future research.

\section{References}

Anderson, S. P., \& Glomm, G. (1992). Incumbency effects in political campaigns. Public Choice, 74(2), 207-219.

Anderson, S. P., Goeree, J. K., \& Ramer, R. (1997). Location, location, location. Journal of Economic Theory, 77(1), 102-127.

Armstrong, M. (2006). Competition in two-sided markets. The RAND Journal of Economics, 37(3), $668-691$.

Barreda-Tarrazona, I., Garca-Gallego, A., Georgantzs, N., Andaluz-Funcia, J., \& Gil-Sanz, A. (2011). An experiment on spatial competition with endogenous pricing. International Journal of Industrial Organization, 29(1), 74-83. (Special Issue: Experiments in Industrial Organization).

Black, D. (1948). On the rationale of group decision-making. Journal of Political Economy, 56(1), 23-34.

Böckem, S. (1994). A generalized model of horizontal product differentiation. The Journal of Industrial Economics, 42(3), 287-298.

Buechel, B., \& Roehl, N. (2015). Robust equilibria in location games. European Journal of Operational Research, 240(2), 505-517.

Congleton, R. (2002). The median voter model. In F. Rowley \& R. K. Schneider (Eds.), The encyclopedia of public choice. Berlin: Kluwer Academic Publishers.

d'Aspremont, C., Gabszewicz, J. J., \& Thisse, J.-F. (1979). On Hotelling's "stability in competition". Econometrica, 47(5), 1145-1150.

Datta, S., \& Sudhir, K. (2013). Does reducing spatial differentiation increase product differentiation? Effects of zoning on retail entry and format variety. Quantitative Marketing \& Economics, 11(1), 83-116.

De Palma, A., Ginsburgh, V., Papageorgiou, Y. Y., \& Thisse, J. F. (1985). The principle of minimum differentiation holds under sufficient heterogeneity. Econometrica, 53(4), 767-781.

Demange, G. (1982). Single-peaked orders on a tree. Mathematical Social Sciences, 3(4), 389-396. 
Dixon, H. (1987). Approximate Bertrand equilibria in a replicated industry. Review of Economic Studies, 54(177), 47-62.

Downs, A. (1957a). An economic theory of democracy. New York: Harper.

Downs, A. (1957b). An economic theory of political action in a democracy. Journal of Political Economy, 65(2), 135-150.

Eaton, B. C., \& Lipsey, R. G. (1975). The principle of minimum differentiation reconsidered: Some new developments in the theory of spatial competition. The Review of Economic Studies, 42(1), $27-49$.

Economides, N. (1986). Minimal and maximal product differentiation in Hotelling's duopoly. Economics Letters, 21(1), 67-71.

Gal-Or, E., \& Dukes, A. (2003). Minimum differentiation in commercial media markets. Journal of Economics \& Management Strategy, 12(3), 291-325.

George, L. M., \& Waldfogel, J. (2006). The New York Times and the market for local newspapers. American Economic Review, 96(1), 435-447.

Hauser, J. R. (1988). Competitive price and positioning strategies. Marketing Science, 7(1), 76-91.

Hehenkamp, B., \& Wambach, A. (2010). Survival at the center-the stability of minimum differentiation. Journal of Economic Behavior \& Organization, 76(3), 853-858.

Hotelling, H. (1929). Stability in competition. The Economic Journal, 39(153), 41-57.

Hummel, P. (2010). Flip-flopping from primaries to general elections. Journal of Public Economics, 94(11-12), 1020-1027.

Irmen, A., \& Thisse, J.-F. (1998). Competition in multi-characteristics spaces: Hotelling was almost right. Journal of Economic Theory, 78(1), 76-102.

Koutsoupias, E., \& Papadimitriou, C. H. (2009). Worst-case equilibria. Computer Science Review, 3(2), 65-69.

Król, M. (2012). Product differentiation decisions under ambiguous consumer demand and pessimistic expectations. International Journal of Industrial Organization, 30(6), 593-604.

Loertscher, S., \& Muehlheusser, G. (2011). Sequential location games. RAND Journal of Economics, 42(4), 639-663.

Meagher, K. J., \& Zauner, K. G. (2004). Product differentiation and location decisions under demand uncertainty. Journal of Economic Theory, 117(2), 201-216.

Nehring, K., \& Puppe, C. (2007). The structure of strategy-proof social choice-part i: General characterization and possibility results on median spaces. Journal of Economic Theory, 135(1), 269-305.

Osborne, M. J., \& Pitchik, C. (1987). Equilibrium in Hotelling's model of spatial competition. Econometrica: Journal of the Econometric Society, 55(4), 911-922.

Panzar, J. C. (1979). Equilibrium and welfare in unregulated airline markets. American Economic Review, 69(2), 92-95.

Prescott, E. C., \& Visscher, M. (1977). Sequential location among firms with foresight. Bell Journal of Economics, 8(2), 378-393.

Radner, R. (1980). Collusive behavior in noncooperative epsilon-equilibria of oligopolies with long but finite lives. Journal of Economic Theory, 22(2), 136-154.

Rhee, B.-D., Palma, A., Fornell, C., \& Thisse, J.-F. (1992). Restoring the principle of minimum differentiation in product positioning. Journal of Economics \& Management Strategy, 1(3), 475-505.

Rochet, J.-C., \& Tirole, J. (2003). Platform competition in two-sided markets. Journal of the European Economic Association, 1(4), 990-1029.

Samuelson, L. (1984). Electoral equilibria with restricted strategies. Public Choice, 43(3), 307-327.

Schipper, Y., Nijkamp, P., \& Rietveld, P. (2007). Deregulation and welfare in airline markets: An analysis of frequency equilibria. European Journal of Operational Research, 178(1), 194-206.

Stern, L. W., Agodo, O., \& Firat, F. A. (1976). Territorial restrictions in distribution: A case analysis. Journal of Marketing, 40(2), 69-75.

Tabuchi, T. (2012). Multiproduct firms in Hotelling's spatial competition. Journal of Economics \& Management Strategy, 21(2), 445-467.

Tabuchi, T., \& Thisse, J.-F. (1995). Asymmetric equilibria in spatial competition. International Journal of Industrial Organization, 13(2), 213-227.

Waldfogel, J. (2008). The median voter and the median consumer: Local private goods and population composition. Journal of Urban Economics, 63(2), 567-582.

Xefteris, D. (2013). Hotelling was right. Journal of Economics \& Management Strategy, 22(4), 706-712. 